\title{
Space/Time Traffic Fluctuations in a Cellular Network: Measurements' Analysis and Potential Applications
}

\author{
Juan Sánchez-González, Oriol Sallent, Jordi Pérez-Romero \\ Universitat Politècnica de Catalunya (UPC), c/ Jordi Girona, 1-3, 08034 Barcelona, Spain \\ juansanchez@tsc.upc.edu
}

\begin{abstract}
The characterization of the space/time traffic profiles in a cellular network can be of high interest for automating the operation of future networks, since the knowledge extracted from the traffic fluctuations in a cell and its neighbours can be effectively exploited by different optimisation functions. In this context, this paper takes as an input a set of real traffic measurements in a cellular network deployed in a large city and analyses, on a per cell basis, the traffic profile characteristics at different time scales (week, day, hour). Then, the analysis is extended to the space dimension by considering the traffic of one cell in relation to that of its neighbours. This allows identifying traffic complementarities between neighbour cells at different time scales that can be exploited by certain optimisation functions, as illustrated in the paper with specific examples.
\end{abstract}

Keywords: Space/time traffic analysis, cellular networks, SON, Mobility Load Balancing, Coverage and Capacity Optimisation, energy saving.

\section{Introduction}

In the last years, cellular networks have witnessed an explosive increase in the traffic volume due to the massive penetration of new wireless devices (such as smartphones and tablets). It is envisaged that this trend will continue in the future with the emergence of new bandwidth-intensive applications such as high definition video, 3D, augmented reality/virtual reality, etc. and with the demand for wireless Machine-toMachine communications. According to [1], the worldwide mobile devices are expected to consume more than 48.3 Exabytes per month by 2021, which represents a sevenfold increase between 2016 and 2021. To cope with these challenging requirements, research and standardization work is nowadays focused on the development of the 5th Generation $(5 \mathrm{G})$.

$5 \mathrm{G}$ networks are expected to be characterized by unprecedented flexibility to support a wide range of requirements and network functionalities associated to different application scenarios, going beyond the provision of enhanced Mobile BroadBand (eMBB) services to incorporate services associated to other sectors such as automotive, utilities, smart cities, etc. The resulting heterogeneity and the required flexibility 
will make the network planning and operation much more complex, thus claiming for an increase in the degree of automation of these processes.

Legacy systems such as $2 \mathrm{G} / 3 \mathrm{G} / 4 \mathrm{G}$ already started the path towards a higher degree of automation through the introduction of Self-Organizing Network (SON) functionalities [2]. However, nowadays, the volume and variety of measurement data that can be obtained from the mobile network is overwhelming. Due to the recent developments of big data technologies [3], it is envisioned that 5G SON will better exploit the huge amount of data available by a Mobile Network Operator (MNO) [4][5]. The target is to efficiently handle this big amount of data and turn it into value by gaining insight and understanding data structures and relationships, extracting exploitable knowledge and deriving successful decision-making.

Obtaining knowledge of space/time traffic profiles can be of high interest for network optimisation since the analysis of traffic spatio/temporal fluctuations in a given cell and its neighbours can be effectively exploited by different decision making processes associated to the planning and operation of the network. In this direction, several works have proposed methodologies to analyse the base stations traffic to obtain knowledge of the network usage to be used for network optimization. As an example, [6] provides a geospatial and temporal characterization of application usage in a real $3 \mathrm{G}$ cellular data network. In particular, the paper studies where and when users are running the different services (e-mail, web-browsing, etc.) in order to improve the radio resource provision in the network. Similarly, [7] proposes a methodology to identify different types of cells (i.e. cells deployed in residential areas, commercial areas, etc.) by evaluating several features such as traffic fluctuation, user mobility, temporal pattern of traffic generation and user service preferences.

Some works, such as [8][9], make use of real measurements to extract spatiotemporal traffic models that are used for traffic forecasting. The characterization and modelling of spatio-temporal fluctuations in the access network by analyzing real measurements in cellular networks has been addressed by [10][11][12]. In particular, according to [11], the spatial distribution of traffic can be assumed to be log-normal distributed around certain geographical areas with high traffic demands. However, the temporal evolution of traffic can be represented as the summation of different sinusoids with periods of 8,12 and 24 hours to capture the user social behavior, because mobile users tend to have repetitive behaviors in periods of one day, half a day and working hours. Some works make use of Artificial Intelligence mechanisms in order to extract knowledge of the time traffic evolution. As an example, [13] compares Support Vector Machine and K-means methodologies for the classification of cells with high load levels during all the day, cells with a peak load level in the morning and cells with a peak load level in the evening. Classification algorithms are used in [14] to identify cells with periods of low traffic level and cells with peaks of traffic and apply this knowledge in different management processes such as energy saving or spectrum planning. Similarly, [15] proposes an algorithm that exploits knowledge of spatio-temporal traffic fluctuations to save energy by switching off certain base stations without reducing the network capacity.

While the above papers have addressed different strategies to analyze the traffic in the different cells, none of the previous works have focused on the identification of spatio-temporal traffic complementarities among neighbor cells and how to exploit 
them for optimizing the operation of the network. In this context, this paper provides two main contributions. First, it presents a statistical analysis of a bunch of realistic traffic measurements obtained from hundreds of cells in a large city to evaluate at what extent fluctuations exist in real traffic when considering, on a per cell basis, the traffic profile characteristics at different time scales (week, day, hour). Second, the analysis is extended to incorporate the spatial dimension by illustrating, through several examples, some existing traffic complementarities between neighbour cells at different time scales. For each example, the paper discusses possible network reconfiguration actions that can make use of the identified complementarities.

The rest of the paper is organised as follows. Section 2 presents the statistical analysis of the real traffic measurements in the time domain, while Section 3 presents examples of traffic complementarities between cells and possible ways to exploit them for the optimisation of the radio resource usage. Finally, Section 4 summarizes the main conclusions.

\section{Analysis of traffic measurements in time}

The available measurements have been obtained from 423 cells that belong to a real cellular network deployed in the city centre of one of the major cities in Spain. For each cell, the measurements have been recorded during one week in periods of 15 minutes. For the analysis of the time evolution of the cell traffic, different averaging periods (i.e. 2, 8 and 24 hours) have been considered. Due to confidentiality reasons, the presented traffic load has been normalised. In any case, this normalisation does not affect the analysis of the results, since we are only interested in relative traffic variations between cells.

Considering the measurements of all the cells, Fig. 1 shows the $10^{\text {th }}, 50^{\text {th }}$ and $90^{\text {th }}$ percentile of the normalised average daily traffic from Monday to Sunday (i.e. in the presented results, $\mathrm{X}^{\text {th }}$ percentile represents the average daily traffic that is not exceeded by $\mathrm{X} \%$ of the cells). It is observed that, over 24 hours period (i.e., on a per-day view) and over the whole scenario, traffic keeps at a rather constant level during weekdays and lowers during weekends. Nevertheless, the statistical analysis made at a shorter time scale provides further knowledge. As an example, Fig. 2 shows the $10^{\text {th }}$,

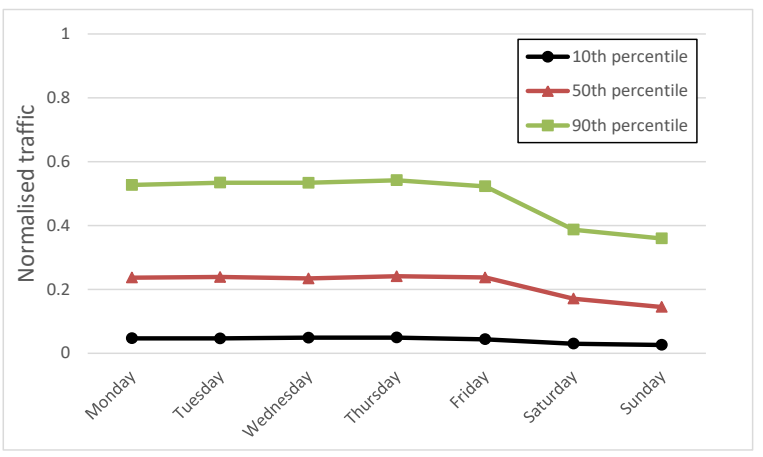

Fig. 1. $10^{\text {th }}, 50^{\text {th }}$ and $90^{\text {th }}$ percentile of the average daily traffic. 


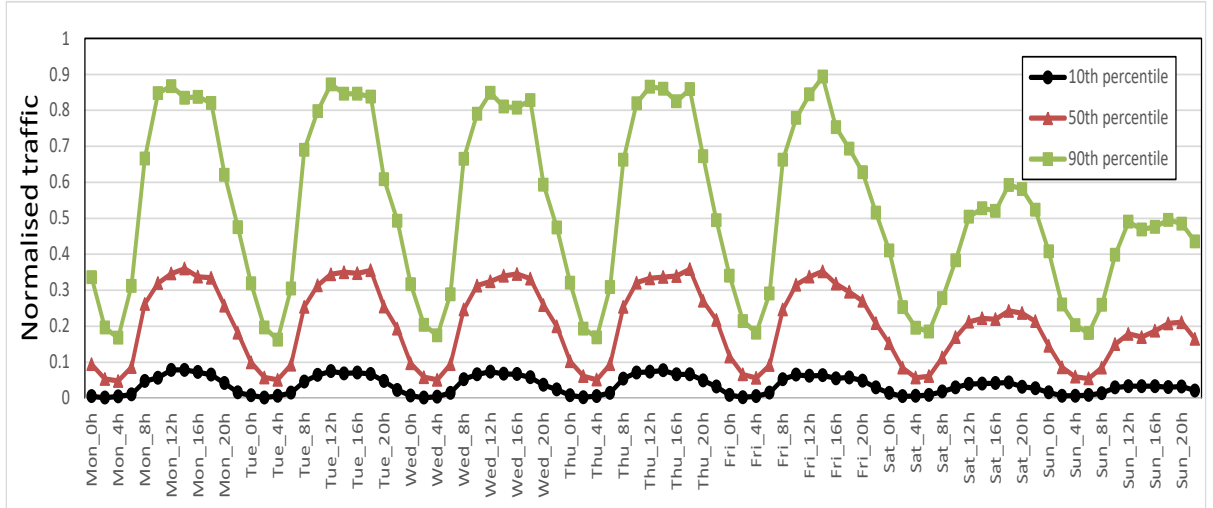

Fig. 2. $10^{\text {th }}, 50^{\text {th }}$ and $90^{\text {th }}$ percentile of the average traffic over $2 \mathrm{~h}$ periods.

$50^{\text {th }}$ and $90^{\text {th }}$ percentile of the average traffic over 2 hours periods and over the whole scenario from Monday to Sunday. This view allows observing intra-day traffic variations, exhibiting higher traffic levels during working hours (i.e., from 8AM to 6PM).

In order to assess at what extent the different cells exhibit different traffic behaviours, the average daily traffic per cell from Monday to Friday is analysed. For each day, each cell is classified as having low (L), medium (M) or high (H) traffic. Based on this, it is found that $84 \%$ of the cells are classified in the same category (L, M or $\mathrm{H})$ every day from Monday to Friday. Therefore, the other $16 \%$ of the cells exhibit significant daily traffic variations during weekdays. Fig. 3 depicts some examples of these cells. For example, Cell 264 has L traffic from Monday to Thursday and M traffic on Friday. In turn, Cell_93 has only $\mathrm{H}$ traffic on Thursday. In some cases, a reason to explain the traffic variation from one day to another can be found (e.g., Cell_93 is located in an area where there is a weekly street market on Thursday), while in other cases the source of the traffic fluctuation remains unclear.

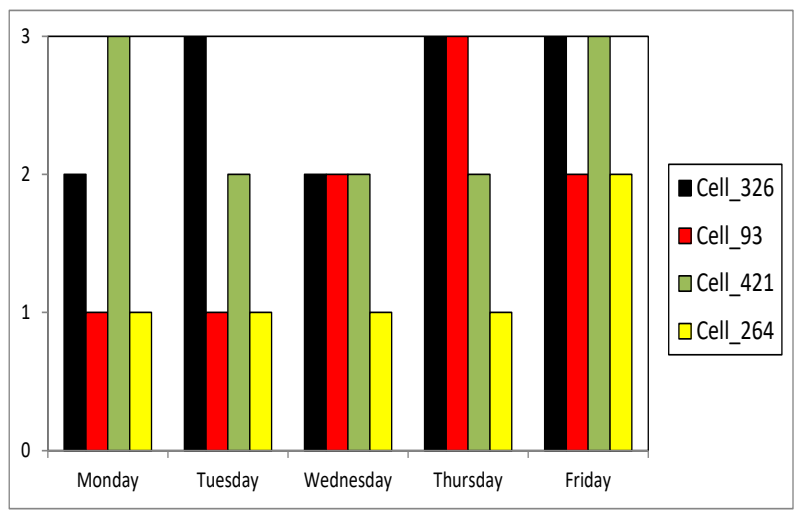

Fig. 3. Traffic level evolution during weekdays. 
The average traffic over periods of 8 hours (i.e. $0 \mathrm{~h}-8 \mathrm{~h}, 8 \mathrm{~h}-16 \mathrm{~h}$ and $16 \mathrm{~h}-24 \mathrm{~h}$ ) has been also analysed from Monday to Friday. Again, for each period of 8 hours, each cell is classified as having L, M or $\mathrm{H}$ traffic. Table 1 presents the percentage of cells classified in the same category every day from Monday to Friday and the percentage of cells classified differently in some days. It is observed that, at night, around $89 \%$ of the cells are classified equally (i.e. with L traffic) all days while more differences are observed in the evening.

Table 1. Percentage of cells classified under the same and under different categories in all the days from Monday to Friday.

\begin{tabular}{|l|l|l|}
\hline Period & $\begin{array}{l}\text { \% of cells with the same traffic } \\
\text { level (H, M or L) in all days }\end{array}$ & $\begin{array}{l}\text { \% of cells with different traf- } \\
\text { fic levels in different days }\end{array}$ \\
\hline Night (0h-8h) & $89 \%$ & $11 \%$ \\
\hline Morning (8h-16h) & $83 \%$ & $17 \%$ \\
\hline Evening (16h-24h) & $74 \%$ & $26 \%$ \\
\hline
\end{tabular}

Fig. 4a and Fig. 4b show examples of cells with different classification on weekdays during the periods $8 \mathrm{~h}-16 \mathrm{~h}$ and $16 \mathrm{~h}-24 \mathrm{~h}$, respectively. Note, for example, that Cell_264 has L traffic from Monday to Thursday while it has M traffic on Friday morning and $\mathrm{H}$ traffic on Friday evening. The reason of this behaviour is that Cell_264 is located in one of the most important entertainment and shopping areas of the city where the population density is higher especially on Friday evening. In turn, Cell 93 has a peak traffic on Thursday morning, while the traffic is M or L for the rest of the $8 \mathrm{~h}$ periods of the week. The $\mathrm{H}$ traffic on Thursday was already identified in Fig. 3. A closer look allowed identifying that the $\mathrm{H}$ traffic is mostly during the working hours, as the traffic increase is due to the presence of the weekly street market.

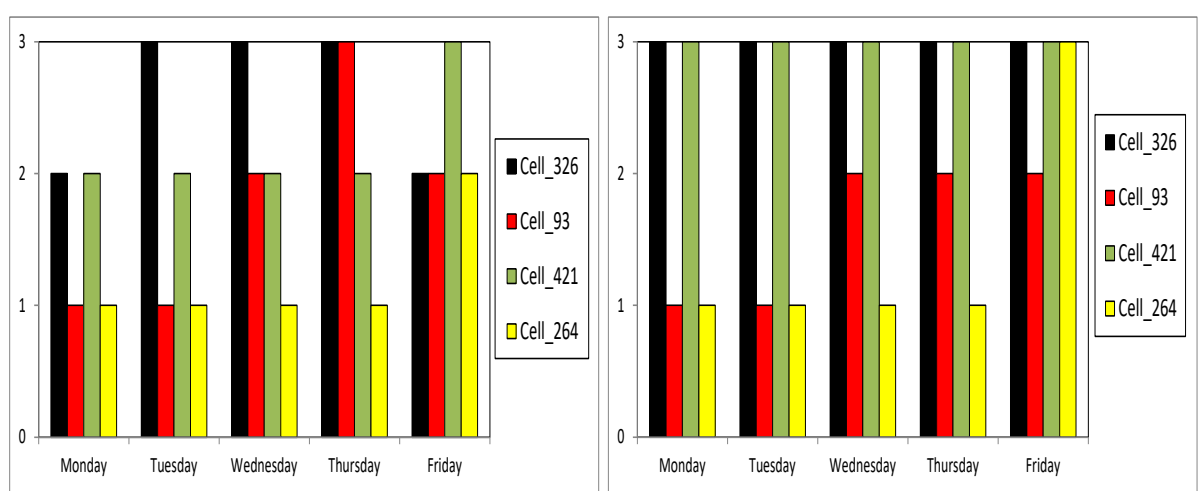

(a)

(b)

Fig. 4. Traffic level during weekdays: a) in the period $8 \mathrm{~h}-16 \mathrm{~h}$; b) in the period $16 \mathrm{~h}-24 \mathrm{~h}$ 


\section{Analysis of traffic measurements in time and space}

Obtaining knowledge of spatio-temporal traffic fluctuations and traffic complementarities between neighbour cells will clearly be very valuable for the optimisation of the radio resource usage in the different cells. Specifically, the identification of traffic complementarities can be helpful as an input for certain SON functions that automate the operation of the network (see e.g. [2][16] for background reading about SON functions). In this respect, both self-optimization functions, which intend to tune certain network parameters according to specific performance targets, and selfplanning functions, which intend to automate the decisions of deploying new nodes in the network, can benefit from the analysis of traffic in the different cells and the identification of traffic complementarities. Depending on the time scale of the discovered complementarities, different kind of actions over the network may be done.

In the following, some examples of traffic complementarities found in the analysed scenario are presented and possible network reconfiguration actions are discussed.

\subsection{Case study \#1}

Fig. 5a presents a region with the geographical location of some cells of the network, while Fig. 5b shows the evolution of the daily and 2-hour averaged traffic during the week in Cell_119 and Cell_1. It is observed that Cell_119 presents a high level of traffic during morning and evening and lower traffic values at night. In turn, Cell_1 is located in an area with lower population density and, consequently, it has lower traffic levels. If the traffic was analysed separately on a per-cell basis, the high traffic level experienced by Cell_119 would suggest a planning decision involving the deployment of a new cell in its proximity or an increase in the capacity of Cell_119, e.g. by deploying a new carrier. Nevertheless, if the analysis considers the complementarities

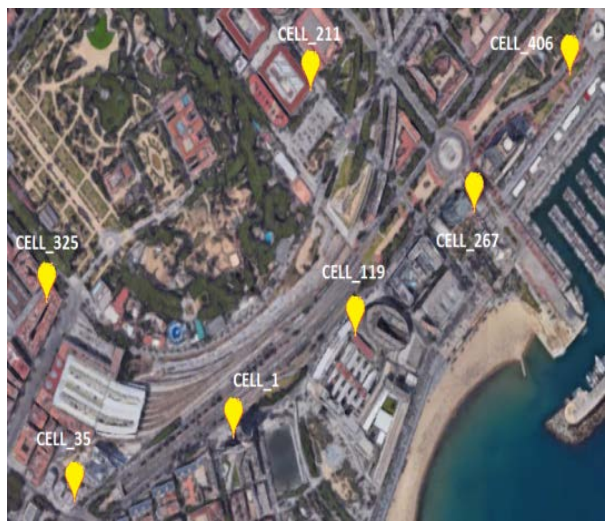

(a)

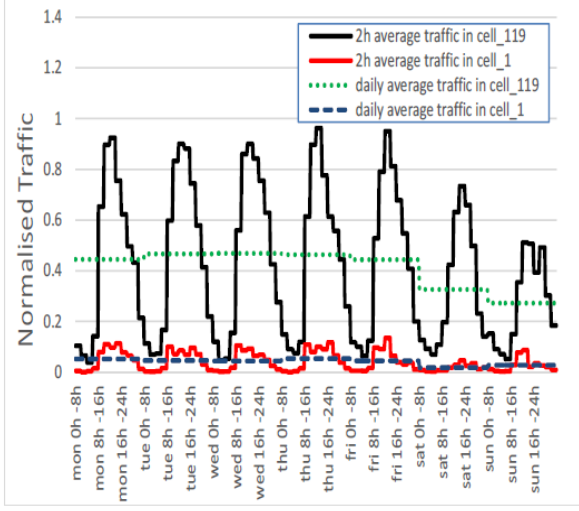

(b)

Fig. 5. (a) Considered scenario; (b) Normalised daily and 2-hour averaged traffic for Cell_1 and Cell_119. 
among neighbour cells, it is possible to see in Fig. $5 \mathrm{~b}$, that the average traffic of neighbour Cell 1 is substantially lower than that of Cell_119 in all the considered time scales. As a result, the self-planning function can identify that no new cell is actually needed because the excess of traffic in Cell_119 can be absorbed by Cell_1. To achieve this, the network can rely on either the Coverage and Capacity Optimization (CCO) or the Mobility Load Balancing (MLB) self-optimization functions. Specifically, the $\mathrm{CCO}$ function can adjust the antenna tilts of the involved cells so that their coverage areas fit better their traffic demands. For example, the CCO function can decide an increase in the antenna tilt of Cell_119 and a reduction of the tilt of Cell_1. This would allow that more users in the area will tend to connect to Cell_1 and would reduce the overload situations of Cell_119. Alternatively, a similar effect can be obtained through the MLB function, which would adjust the cell reselection and handover thresholds to force that more users in the area connect through the less loaded Cell_1.

\subsection{Case study \#2}

Fig. 6a presents the geographical location of several cells in an area in the city centre, while Fig. 6b shows the weekly and daily average traffic for Cell_202 and Cell_265. Although the weekly average traffic in both cells is very similar, the daily average traffic shows some complementarities between these two cells. As shown in Fig. 6b, Cell 265 has a relatively high load level on Friday and Saturday since this cell is located in an important shopping and entertainment street in the city, where people usually go shopping in the weekend. On the other hand, Cell_202 has higher traffic levels during the weekdays since it is located in a nearby business street, where people usually stay during working hours. Based on this, the system could rely on the CCO or MLB functions. For example, two different configurations could be considered for setting the antenna tilts and better adapt to the different traffic demands. On

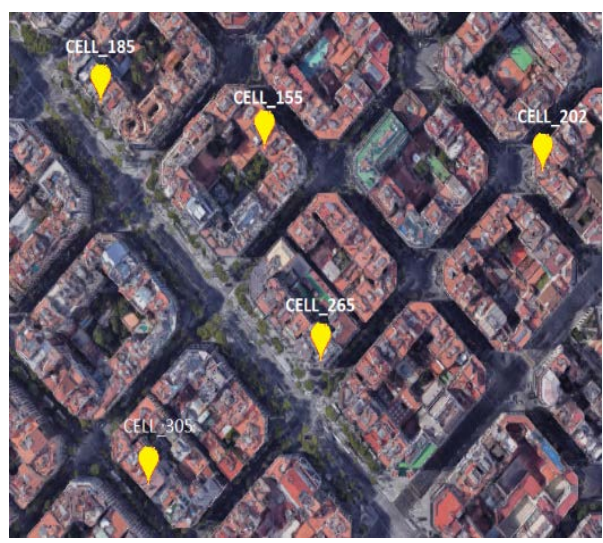

(a)

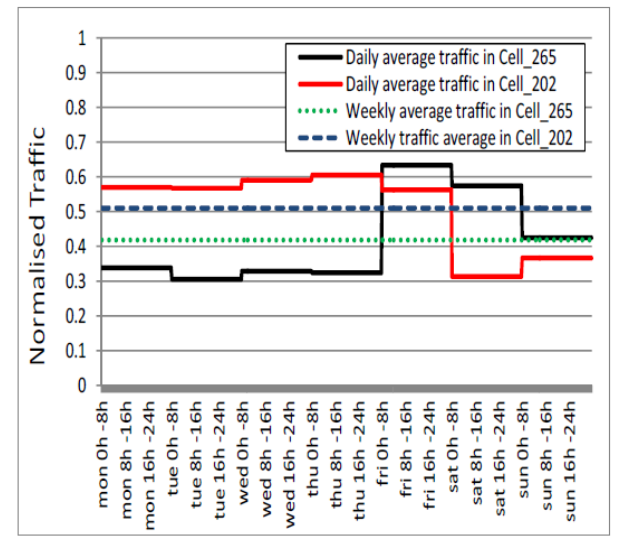

(b)

Fig. 6. (a) Considered scenario; (b) Normalised weekly and daily averaged traffic for Cell_202 and Cell_265. 
Friday and Saturday the selected configuration would consist in a higher tilt in Cell 265 and a lower one in Cell 202. This would allow shedding traffic from Cell 265 to Cell 202. For the rest of the days, the configuration would consist in a lower tilt in Cell_265 and a higher one in Cell_202 in order to shed traffic from Cell_202 to Cell_265.

\subsection{Case study \#3}

Fig. 7a shows the geographical locations of Cell_36 and Cell_49, while Fig. 7b presents the normalised daily and 8-hour average traffic in both cells. Cell_36 is deployed in an entertainment area of the city with plenty of restaurants and shops, while Cell_49 provides coverage to some office buildings located in the harbour area. Although the daily average traffic from Monday to Thursday is very similar in Cell_36 and Cell 49, the traffic averaged over periods of 8 hours reveals that there is a clear complementarity between the morning and evening traffic served by Cell 36 and Cell_49 in the weekdays. In particular, Cell_49 exhibits a substantially high traffic during mornings, while at the same time the traffic in Cell_36 is lower. The situation reverses during evenings and nights and during the weekend, when the traffic in Cell_36 is higher than that of Cell_49. The identification of this complementarity suggests that the high traffic situations in either one or the other cell could be solved by shifting traffic from the highly loaded cell to the less loaded one. Again, this can be achieved through the CCO/MLB functions, so that traffic would be shed from Cell_49 to Cell_36 in the morning while traffic would be shed from Cell_36 to Cell_49 in the evening and during the weekends.

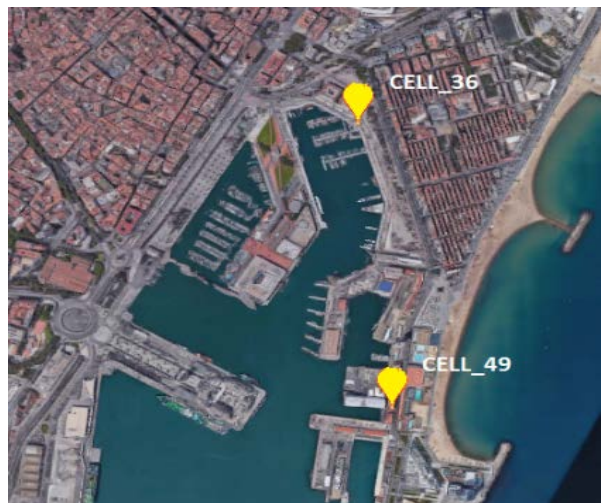

(a)

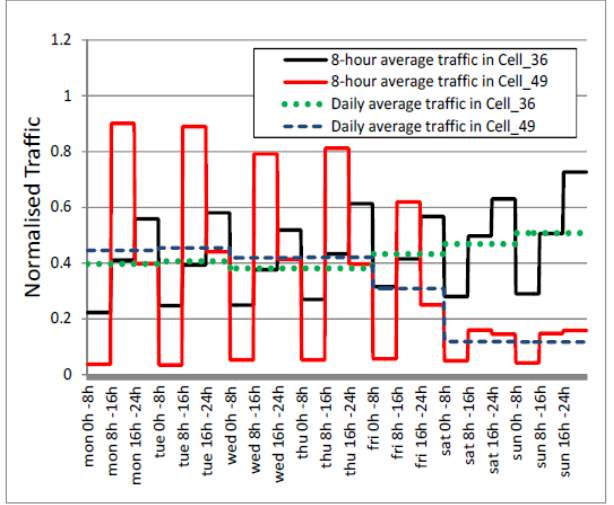

(b)

Fig. 7. (a) Considered scenario; (b) Normalised daily and 8-hour averaged traffic for Cell_36 and Cell_49.

\subsection{Case study \#4}

Fig. 8a presents the geographical location of several cells in another area of the city center, while Fig. 8b presents the daily and 8-hour average traffic in Cell_111 and 
Cell 286. It is worth noting that there are periods of 8-hours where the average traffic is very low in Cell 111 (especially at night and in the weekend). Based on this, the energy saving self-optimization function can identify Cell_111 as a candidate cell to be switch off during these periods of time and serve this traffic from a neighbor cell (i.e. Cell_286 in this example).

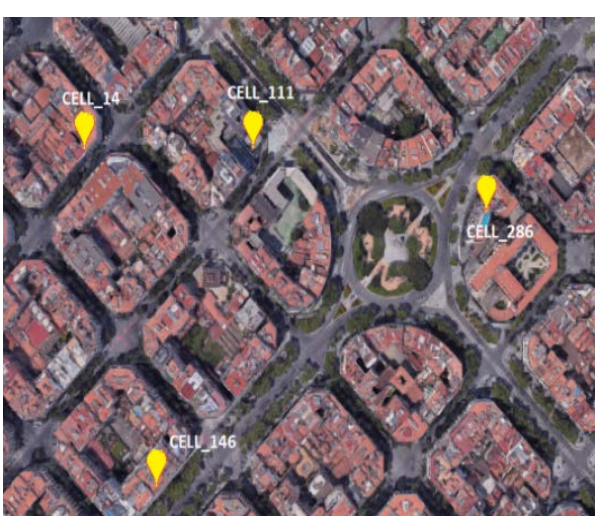

(a)

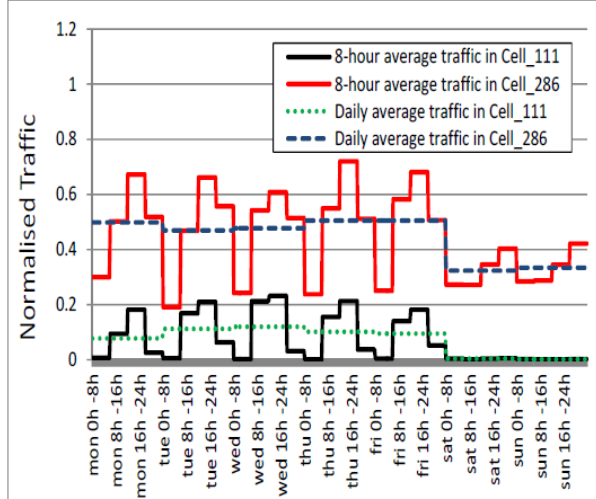

(b)

Fig. 8. (a) Considered scenario; (b) Normalised daily and 8-hour averaged traffic for Cell_111 and Cell_286.

\section{Conclusions}

This paper has presented a statistical analysis of spatio-temporal traffic fluctuations and complementarities among neighbour cells by processing real measurements obtained from hundreds of cells deployed in a large city. Besides well expected observations, such as that the average traffic per day keeps at a rather constant level during weekdays and that higher traffic levels are observed during working hours in case of shorter averaging periods (e.g. $2 \mathrm{~h}, 8 \mathrm{~h}$ ), the measurements have revealed that around $16 \%$ of the studied cells present significant inter-day traffic variations during weekdays. In some cases, an explanation can be found (e.g. a street market that occurs on a specific day of the week in the area of the cell, thus causing a traffic increase on that day), while in other cases the explanation remains unknown.

The analysis has also jointly considered the traffic of a cell and its neighbours. This study has allowed the identification of some examples of areas exhibiting traffic complementarities among cells at different time scales. The detection of these complementarities can be valuable for supporting the operation of certain SON functions and optimizing the behaviour of the network. In particular, the presented examples illustrate situations in which the CCO/MLB functions can adjust the tilt or the handover and cell reselection thresholds to shift traffic from a highly loaded cell to a less loaded neighbour. The application of these actions avoids having to deploy a new cell to cope with the high load situations. Similarly, another example has illustrated the possibility of exploiting the traffic complementarities for energy saving purposes, identifying cells that can be switched-off during night periods. 
Acknowledgements. This work has been supported by the EU funded H2020 5G-PPP project 5G ESSENCE under grant agreement 761592 and by the Spanish Research Council and FEDER funds under RAMSES and SONAR 5G grants (ref. TEC201341698-R and TEC2017-82651-R).

\section{References}

1. Cisco Visual Networking Index: Forecast and Methodology, 2016-2021, White Paper, June 2017.

2. J. Ramiro, K. Hamied, Self-Organizing Networks. Self-planning, self-optimization and self-healing for GSM, UMTS and LTE, John Wiley \& Sons, 2012.

3. Y. Liu, S. Han, S. Wang, G. Liu, "On big data analytics for greener and softer RAN"; IEEE Access, August 2015.

4. A. Imran, A. Zoha, A. Abu-Dayya, "Challenges in 5G: How to empower SON with big data for enabling 5G”, IEEE Network, December 2014, pp. 27-33.

5. J. Pérez-Romero, O. Sallent, R. Ferrús, R. Agustí, “Artificial Intelligence-based 5G Network capacity planning and operation", International Symposium on Wireless Communication Systems (ISWCS 2015), Brussels, Belgium, August 2015.

6. M. Zubair, L. Ji, A.X. Liu, J. Pang, J. Wang, "Geospatial and temporal dynamics of application usage in cellular data networks", IEEE Transactions on mobile computing, Vol. 14, No. 7, July 2015.

7. J. Ma, W. Ni, J. Yin, S. Lin, H. Cui, R.P. Liu, B. X. Fang, "Modelling social characteristics of mobile radio networks", International Conference on Communications (ICC 2015), London, United Kingdom, June 2015.

8. D. Miao, W. Sun, X. Qin, W. Wang, "MSFS: Multiple Spatio-Temporal Scales Traffic Forecasting in Mobile Cellular Network", 14th International Conference on Pervasive Intelligence and Computing, Auckland, New Zealand, August 2016.

9. F. Xiu, Y. Lin, J. Huang, D. Wu, H. Shi, J. Song, Y. Li, "Big Data Drive Mobile Traffic Understanding and Forecasting: A Time Series Approach", IEEE Transactions on Services Computing, Vol 9, No 5, October 2016.

10. D. Lee, S. Zhou, X. Zhong, Z. Niu, "Spatial Modeling of the Traffic Density in Cellular Networks", IEEE Wireless Communications, Vol. 21 Issue 1, pp: 80-88, February 2014.

11. S. Wang, X. Zhang, J. Zhang, J. Feng, W. Wang, K. Xin, "An Approach for Spatialtemporal Traffic Modeling in Mobile Cellular Networks", International Teletraffic Congress (ITC), Ghent, Belgium, September 2015.

12. R. Li, Z. Zhao, X. Zhou, J. Palicot, H. Zhang, "The prediction analysis of cellular radio access network traffic: From entropy theory to networking practice", IEEE Communications Magazine Vol 52 No 6, pp:234-240, 2014.

13. S. E. Hammami, H. Afifi, M. Marot, V. Gauthier, "Network planning tool based on network classification and load prediction", IEEE Wireless Communications and Networking Conference (WCNC 2016), Doha, Qatar, April 2016.

14. J. Pérez-Romero, J. Sánchez-González, O. Sallent, R. Agustí, "On Learning and Exploiting Time Domain Traffic Patterns in Cellular Radio Access Networks", 12th Int. Conf. on Machine Learning and Data Mining (MLDM) 2016, New York, USA, July, 2016.

15. J. Kim, H. W. Lee, S. Chong, "Traffic-aware energy-saving base station sleeping and clustering in cooperative networks", IEEE Trans. on Wireless Comm., Vol: pp, issue 99, 2017.

16. O. G. Aliu, A. Imran, M. A. Imran, B. Evans, "A Survey of Self Organisation in Future Cellular Networks", IEEE Comm. Surveys and Tutorials, Vol. 15, No. 1, November, 2013. 